\title{
Effect of oestrogen plus progestin on the incidence of diabetes in postmenopausal women: results from the Women's Health Initiative Hormone Trial
}

\author{
K. L. Margolis ${ }^{1,9}$ • D. E. Bonds ${ }^{2}$ R. J. Rodabough ${ }^{3}$ L. Tinker ${ }^{3}$ - L. S. Phillips ${ }^{4}$ C. Allen ${ }^{5}$ T. Bassford ${ }^{6}$ \\ G. Burke $^{2} \cdot$ J. Torrens $^{7} \cdot$ B. V. Howard ${ }^{8}$ for the Women's Health Initiative Investigators \\ ${ }^{1}$ Hennepin County Medical Center, Minneapolis, Minnesota, USA \\ ${ }^{2}$ Wake Forest University School of Medicine, Winston-Salem, North Carolina, USA \\ ${ }^{3}$ Fred Hutchinson Cancer Research Center, Seattle, Washington, USA \\ ${ }^{4}$ Emory University, Atlanta, Georgia, USA \\ 5 University of Wisconsin, Madison, Wisconsin, USA \\ ${ }^{6}$ University of Arizona, Tucson, Arizona, USA \\ ${ }^{7}$ University of Medicine and Dentistry of New Jersey, Newark, New Jersey, USA \\ ${ }^{8}$ MedStar Research Institute/Howard University, Washington, District of Columbia, USA \\ ${ }_{9}^{9}$ Berman Center for Outcomes and Clinical Research, Minneapolis, USA
}

\begin{abstract}
Aims/hypothesis. Studies examining the effect of postmenopausal hormone therapy on concentrations of glucose, insulin and diabetes incidence have been inconclusive, in part because many of the studies were too small. We examined the effect of oestrogen plus progestin on diabetes incidence and insulin resistance.

Methods. The study was a randomised, double-blind trial comparing the effect of daily $0.625 \mathrm{mg}$ conjugated equine oestrogens plus $2.5 \mathrm{mg}$ medroxyprogesterone acetate with that of placebo during 5.6 years of follow-up. The participants were 15,641 postmenopausal women enrolled in the Women's Health Initiative Hormone Trial. These women were aged 50 to 79 and all had an intact uterus. Diabetes incidence was ascertained by self-report of treatment with insulin or oral hypoglycaemic medication. Fasting glucose, insulin, and lipoproteins were measured in a random sample at baseline and at 1 and 3 years.
\end{abstract}

Results. The cumulative incidence of treated diabetes was $3.5 \%$ in the hormone therapy group and $4.2 \%$ in the placebo group (hazard ratio $0.79,95 \%$ CI $0.67-0.93$, $p=0.004$ ). There was little change in the hazard ratio after adjustment for changes in BMI and waist circumference. During the first year of follow-up, changes in fasting glucose and insulin indicated a significant fall in insulin resistance in actively treated women compared to the control subjects (Year 1 to baseline betweengroup difference $-0.22 \pm 0.10, p=0.03)$.

Interpretations/conclusion. These data suggest that combined therapy with oestrogen and progestin reduces the incidence of diabetes, possibly mediated by a decrease in insulin resistance unrelated to body size. Future studies of alternative postmenopausal hormone therapy regimens and selective oestrogen agonists and/or antagonists should consider the effects of these regimens on insulin resistance and diabetes.

Keywords Diabetes mellitus - Double-blind method · Oestrogens $\cdot$ Postmenopause $\cdot$ Progestins $\cdot$ Random allocation
Received: 12 February 2004 / Accepted: 22 April 2004

Published online: 14 July 2004

(C) Springer-Verlag 2004

\section{K. L. Margolis ( $)$}

Berman Center for Outcomes and Clinical Research, 825 S. 8th Street, Suite 440, Minneapolis, MN 55404, USA E-mail: margo006@umn.edu

Tel.: +1-612-3477756, Fax: +1-612-3417935

\section{Introduction}

Type 2 diabetes is increasingly being recognised as a critical health problem in middle-aged and elderly people [1]. It is therefore important to identify inter-

Abbreviations: HERS, Heart and Estrogen/Progestin Replacement Study · HOMA-IR, homeostasis model assessment of insulin resistance $\cdot \mathrm{HR}$, hazard ratio · MET, metabolic equivalent of the task - SHBG, sex hormone binding globulin - WHI, Women's Health Initiative 
ventions that can decrease the risk of diabetes. Clinical trials conducted primarily in women without diabetes have suggested that postmenopausal hormone therapy has favourable effects on at least some measures of glucose homeostasis and body composition $[2,3,4,5,6,7,8,9,10]$. However, other trials have suggested there is no benefit, or even that carbohydrate metabolism worsens with hormone therapy $[11,12,13,14,15,16,17,18,19,20]$. The latter tended to be older trials or have small sample sizes. Prospective observational studies examining diabetes as an outcome have also been inconclusive [21, 22, 23 , 24], although the largest of these found a $20 \%$ reduction in diabetes risk among current hormone therapy users over 12 years of follow-up [25].

The Heart and Estrogen/Progestin Replacement Study (HERS) recently published data on incident diabetes in 2029 postmenopausal women who had coronary heart disease and had been assigned to daily oestrogen plus progestin or to placebo [26]. Over 4 years, the incidence of diabetes was $6.2 \%$ in the oestrogen plus progestin group and $9.5 \%$ in the placebo group (hazard ratio [HR] $0.65,95 \%$ CI $0.48-0.89$ ). Although the difference could not be attributed to changes in weight or waist circumference, insulin was not measured in this study, precluding analysis of changes in insulin resistance as a possible contributory mechanism.

The Women's Health Initiative (WHI) Hormone Trial was initiated to determine the risks and benefits of hormone therapy on a wide range of important health outcomes in predominantly healthy postmenopausal women. The primary endpoint of the trial was death from coronary heart disease or non-fatal myocardial infarction. Seconday outcomes were: hip fracture, invasive breast cancer, colorectal cancer, stroke, pulmonary embolism, endometrial cancer and death from other causes [27]. The trial was terminated early after an average of 5.6 years of follow-up because of an adverse effect on breast cancer and a global index showing that the overall risks of the intervention exceeded the benefits [28].

In this paper, we compare another aspect of hormone therapy, namely the effect of daily oestrogen plus progestin or placebo on glucose, insulin, calculated insulin resistance, and the self-reported incidence of diabetes over more than 5 years of follow-up.

\section{Subjects and methods}

Study population. Details on eligibility, recruitment methods, study population characteristics, hormone regimens, randomisation, blinding, follow-up and the main outcomes of the WHI Estrogen plus Progestin Trial have been published [27, 28]. Briefly, 16,608 postmenopausal women were recruited at 40 clinical centres throughout the United States. These women were aged 50 to 79, and all of them had an intact uterus. Major exclusion criteria were a history of previous breast cancer, any cancer within the previous 10 years except non-melanoma skin cancer, and current use of corticosteroids, anticoagulants, tamoxifen or other selective oestrogen receptor modifiers. A history of venous thromboembolism was added as an exclusion criterion in 1997 . Women with severe menopause symptoms who were unwilling to discontinue the use of menopausal hormone therapy were excluded, and a 3-month washout period was required for women who were current hormone users at the initial screening visit.

The WHI also includes three other randomised trials: a companion hormone trial of oestrogen alone in women who have had a hysterectomy, a dietary modification trial examining the health outcomes of a low-fat eating pattern, and a trial of calcium and vitamin D. Approximately $29 \%$ of women in the hormone trials are also participating in the dietary modification trial. The protocol and consent forms were approved by the institutional review boards for all participating institutions.

At baseline, participants were asked if a physician had ever told them that they had "sugar diabetes" when they were not pregnant. We considered a positive response to this question a self-reported history of diabetes at baseline, and therefore excluded from this analysis of diabetes incidence 492 participants who had been randomised to oestrogen plus progestin and 475 participants who had been randomised to placebo. Age at diagnosis, history of treatment with oral medications, and past and current treatment with insulin shots were determined by follow-up questions. We did not seek to confirm selfreported diabetes by checking medical records, but participants were instructed to bring all current medications, both prescription and non-prescription, to the baseline WHI clinic visit.

Intervention, randomisation and blinding. Combined oestrogen and progestin was provided in one daily tablet containing conjugated equine oestrogens, $0.625 \mathrm{mg}$, and medroxyprogesterone acetate, $2.5 \mathrm{mg}$ (Prempro, Wyeth Ayerst, Philadelphia, Pa., USA). A matching placebo was provided to the control group. The randomisation procedure was developed at the WHI Clinical Coordinating Center and implemented locally through a distributed study database, using a randomised permuted block algorithm, stratified by clinical centre site and age. Participants, clinic staff, investigators and outcomes adjudicators were blinded to treatment assignment. When required for safety or symptom management, an unblinding officer provided the clinic gynaecologists with the treatment assignment. Neither the clinic gynaecologists nor any of the staff or investigators involved with the clinical care of the participants were involved in assessing the study outcomes.

Some flexibility of the dosages of oestrogen and progestin was allowed to manage symptoms such as breast tenderness and vaginal bleeding. Permanent discontinuation of the study medication was required for women who developed breast cancer, endometrial atypical hyperplasia or cancer, venous thromboembolism, meningioma, malignant melanoma, triglyceride levels greater than $11.3 \mathrm{mmol} / \mathrm{l}$, or were prescribed oestrogen, testosterone, or selective oestrogen receptor modifiers by their personal physicians.

Data collection, follow-up and outcomes ascertainment. Participants attended initial screening visits, during which personal information, medical history, health-related habits, and medication and supplements used were assessed by standardised questionnaires. Diet was assessed using a validated food frequency questionnaire based on instruments previously used in large-scale dietary intervention trials [29, 30]. Recreational physical activity was assessed by questions on the frequency and duration of several types of recreational activity, and metabolic equivalent of the task (MET) scores (defined as the ratio 
of work metabolic rate to a standard resting metabolic rate, with 1 MET roughly equivalent to the resting metabolism while sitting at rest) were computed as the product of days per week, minutes per day, and the MET value for each activity [31]. Height, weight, waist circumference, hip circumference, and blood pressure measurements were obtained by certified staff. Follow-up for clinical events occurred every 6 months, with annual clinic visits required. At annual visits, height, weight and blood pressure measurements were repeated, and adherence to study medication was assessed by pill counts. Waist and hip circumference was measured in all participants at the first annual visit, and in a random sample at the third annual visit. A standardised written protocol, centralised training of local clinic staff, local quality assurance activities, and periodic quality assurance visits by the Clinical Coordinating Center were used to maintain uniform data collection procedures at all study sites.

All women had blood drawn after an overnight 8-hour fast at baseline and at the first annual visit. Serum samples were frozen at $-70{ }^{\circ} \mathrm{C}$ and shipped to the WHI central storage facility. A randomly selected paired $8.6 \%$ sample of blood specimens was analysed at both time points for a set of "core analytes" including glucose, insulin and lipoproteins. The random sampling procedure was stratified by age, clinical centre, hysterectomy status and ethnicity to oversample women from minority populations. The sample cohort also had fasting blood drawn using the same protocol at the third, sixth and ninth annual visits.

At each half-yearly contact, participants were asked the following question: "Since the date given on the front of this form, has a doctor prescribed any of the following pills or treatments?" Choices included "pills for diabetes" and "insulin shots for diabetes". Thus, incident diabetes was defined as a self-reported, new diagnosis of diabetes by a physician, with subsequent treatment with oral hypoglycaemic drugs or insulin.

Statistical analysis. Women with a self-reported history of diabetes at baseline $(n=967,5.8 \%)$ were excluded, with 15,641 women remaining for this analysis. Analyses on stored blood samples were performed on 1362 women at baseline, 1263 women at Year 1 and 1080 women at Year 3. Insulin resistance was calculated from fasting glucose and insulin according to the homeostasis model assessment of insulin resistance (HOMA-IR): insulin $\times($ glucose/22.5), where the units for insulin are $\mathrm{mU} / \mathrm{l}$ and units for glucose are $\mathrm{mmol} / \mathrm{l}$ [32].

Baseline categorical variables were compared either with chi square or Fisher's exact tests for categorical variables or two-sample $t$ tests for continuous variables. Mean differences (at baseline and over time on study) in blood analytes, as well as blood pressure, BMI and waist circumference measures by treatment arm were assessed with $t$ tests. Statistical analyses of blood analyte variables were weighted by race and/or ethnicity. The incidence of diabetes was assessed with a Cox proportional hazards model, stratified by age and dietary modification trial randomisation assignment (not randomised, randomised to intervention group, randomised to control group), in which time to a first self-report of treated diabetes was the outcome variable. The number needed to treat represents the number of women who would need to be treated to prevent one case of diabetes during the average follow-up time, and was calculated as the reciprocal of the absolute difference in the cumulative incidence of diabetes between the intervention and control groups. A sensitivity analysis was performed to account for treatment non-adherence. It was done by comparing KaplanMeier cumulative hazard curves for incidence of diabetes, both for an intention-to-treat analysis, and for participants who were censored 6 months after becoming non-adherent (defined as taking less than $80 \%$ of study medication or starting open-label hormone therapy). All analyses were assessed by comparing $p$ values to a 0.05 level of significance.

Interactions between eight baseline characteristics (age, race/ethnicity, BMI, waist circumference, hypertension, smoking, alcohol use, physical activity) and treatment assignment were assessed in Cox proportional hazards models that included both the risk factor (where applicable, as a continuous variable for computing the $p$ value) and randomisation assignment as main effects. We computed $p$ values for assessing possible interactions from likelihood ratio tests, comparing models with and without the interaction term. Of the eight subgroup comparisons tested, less than one test would be expected to be significant at a $p$ value of 0.05 . Results from all eight comparisons are presented.

Two multivariate age-adjusted Cox proportional hazards models, stratified by dietary modification trial randomisation assignment, were developed to assess treatment effect after adjusting for a one-year change in BMI or waist circumference. After excluding self-reported diabetes in the first year of follow-up, we added tertiles of change in BMI and waist circumference to separate models during the first year of follow-up.

\section{Results}

Of participants who did not report physician-diagnosed diabetes at baseline, 8014 were assigned to receive conjugated equine oestrogens plus medroxyprogesterone acetate and 7627 were assigned to receive placebo. The mean age of the participants was 63 years, $15 \%$ were non-white, and 74\% had never previously used hormone therapy (Table 1). The mean BMI was $28 \mathrm{~kg} / \mathrm{m}^{2}$ and the mean waist circumference was $87 \mathrm{~cm}$. About 19\% were being treated for hypertension and an additional 15\% had blood pressure (average of two seated measurements) at $140 / 90 \mathrm{~mm} \mathrm{Hg}$ or higher at the baseline clinic visit. Of 1359 fasting specimens drawn at baseline and analysed for serum glucose, $70 \%$ were lower than $5.6 \mathrm{mmol} / \mathrm{l}, 27 \%$ were in the range $5.6-6.9 \mathrm{mmol} / \mathrm{l}$, and $3 \%$ were $7.0 \mathrm{mmol} / \mathrm{l}$ or higher. The two treatment groups were well matched with regard to baseline characteristics (Table 1).

There were no differences at baseline between the women in the oestrogen plus progestin and the placebo group with regard to fasting values for glucose, insulin, HOMA-IR, triglycerides, total cholesterol, LDL cholesterol, and HDL cholesterol (Table 2). During the first year of follow-up, glucose fell by $0.12 \mathrm{mmol} / \mathrm{l}$ in the oestrogen plus progestin group, but changed little in the placebo group (Year 1 to baseline between-group difference $-0.11 \pm 0.04 \mathrm{mmol} / 1, p<0.01$ ). In the first year of follow-up, insulin fell modestly in the oestrogen plus progestin group, and increased by a similar amount in the control group (Year 1 to baseline between-group difference $-0.65 \pm 0.34 \mathrm{mU} / 1, p=0.05$ ). These changes in fasting glucose and insulin resulted in a fall in insulin resistance as measured by HOMAIR in actively treated women compared to the control subjects (Year 1 to baseline between-group difference $-0.22 \pm 0.10, p=0.03)$. In the subgroup of 366 women 
Table 1. Baseline characteristics of non-diabetic women randomised to oestrogen plus progesterone or placebo in the Women's Health Initiative

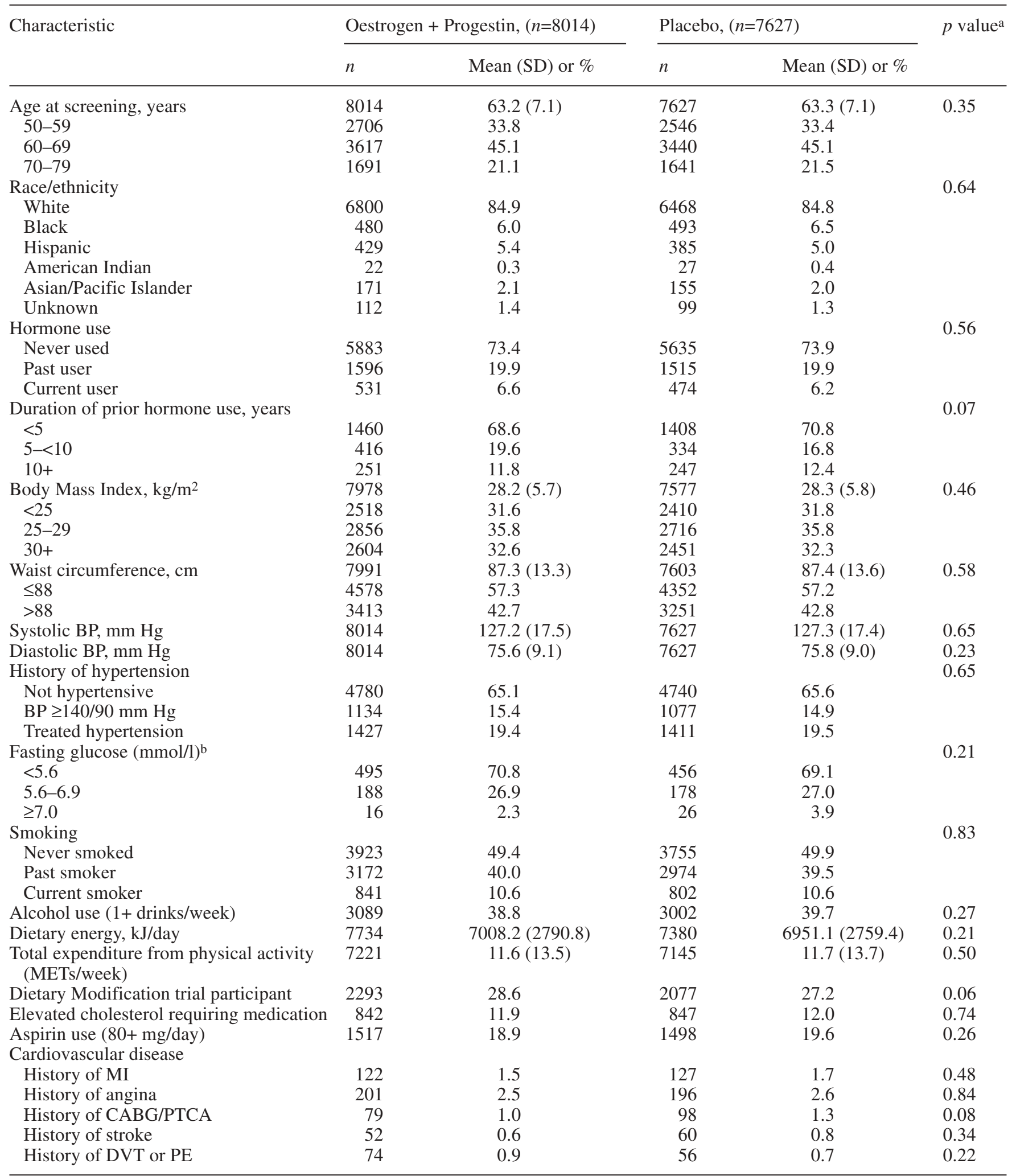

a The $p$ value is from a chi square test or Fisher's exact test for categorical variables and a two-sample $t$ test for continuous variables. ${ }^{b}$ Fasting glucose was only available in an $8.6 \%$ subsample. Abbreviations: BP, blood pressure; MI, myocardial in- farction; CABG/PTCA, coronary artery bypass graft or percutaneous transluminal coronary angioplasty; DVT, deep vein thrombosis; PE, pulmonary embolism 
Table 2. Baseline and follow-up measurements in non-diabetic women randomised to oestrogen plus progesterone or placebo in the Women's Health Initiative ${ }^{a}$

\begin{tabular}{|c|c|c|c|c|c|c|c|c|c|}
\hline & \multicolumn{3}{|c|}{ Oestrogen + Progestin } & \multicolumn{3}{|c|}{ Placebo } & \multirow[t]{2}{*}{ Difference } & \multirow[t]{2}{*}{$(\mathrm{SE})$} & \multirow[t]{2}{*}{$p$ value $^{b}$} \\
\hline & $n$ & Mean & $(\mathrm{SD})$ & $n$ & Mean & (SD) & & & \\
\hline \multicolumn{10}{|l|}{ Glucose (mmol/l) } \\
\hline Baseline & 699 & 5.33 & $(0.71)$ & 660 & 5.35 & $(0.99)$ & -0.02 & $(0.05)$ & 0.63 \\
\hline Year 1 to baseline & 635 & -0.12 & $(0.64)$ & 592 & -0.01 & $(0.70)$ & -0.11 & $(0.04)$ & $<0.01$ \\
\hline Year 3 to baseline & 547 & -0.12 & $(0.62)$ & 507 & -0.04 & $(0.92)$ & -0.08 & $(0.05)$ & 0.15 \\
\hline \multicolumn{10}{|l|}{ Insulin (mU/l) } \\
\hline Baseline & 672 & 11.20 & $(5.65)$ & 643 & 10.63 & $(5.70)$ & 0.57 & $(0.35)$ & 0.11 \\
\hline Year 1 to baseline & 603 & -0.33 & $(5.80)$ & 564 & 0.32 & $(4.27)$ & -0.65 & $(0.34)$ & 0.05 \\
\hline Year 3 to baseline & 496 & 0.76 & $(5.82)$ & 472 & 1.63 & $(6.36)$ & -0.87 & $(0.43)$ & 0.04 \\
\hline \multicolumn{10}{|l|}{ HOMA-IR } \\
\hline Baseline & 672 & 2.73 & (1.59) & 642 & 2.63 & $(1.97)$ & 0.10 & $(0.11)$ & 0.37 \\
\hline Year 1 to baseline & 602 & -0.16 & $(1.48)$ & 562 & 0.05 & $(1.52)$ & -0.22 & $(0.10)$ & 0.03 \\
\hline Year 3 to baseline & 496 & 0.12 & $(1.57)$ & 472 & 0.35 & $(2.00)$ & -0.23 & $(0.13)$ & 0.08 \\
\hline \multicolumn{10}{|c|}{ Triglyceride (mmol/l) } \\
\hline Baseline & 699 & 1.64 & $(0.81)$ & 663 & 1.61 & $(0.87)$ & 0.03 & $(0.05)$ & 0.60 \\
\hline Year 1 to baseline & 635 & 0.07 & $(0.66)$ & 598 & 0.02 & $(0.59)$ & 0.05 & $(0.04)$ & 0.21 \\
\hline Year 3 to baseline & 546 & 0.11 & $(0.75)$ & 507 & 0.05 & $(0.70)$ & 0.05 & $(0.05)$ & 0.26 \\
\hline \multicolumn{10}{|c|}{ Total cholesterol (mmol/l) } \\
\hline Baseline & 699 & 5.84 & $(0.96)$ & 663 & 5.81 & $(0.94)$ & 0.03 & $(0.06)$ & 0.64 \\
\hline Year 1 to baseline & 635 & -0.37 & $(0.74)$ & 598 & -0.04 & $(0.69)$ & -0.34 & $(0.05)$ & $<0.01$ \\
\hline Year 3 to baseline & 546 & -0.35 & $(0.84)$ & 507 & -0.06 & $(0.79)$ & -0.29 & $(0.06)$ & $<0.01$ \\
\hline \multicolumn{10}{|l|}{$\mathrm{LDL}(\mathrm{mmol} / \mathrm{l})$} \\
\hline Baseline & 687 & 3.60 & $(0.87)$ & 652 & 3.59 & $(0.83)$ & 0.01 & $(0.05)$ & 0.92 \\
\hline Year 1 to baseline & 622 & -0.50 & $(0.67)$ & 584 & -0.05 & $(0.58)$ & -0.45 & $(0.04)$ & $<0.01$ \\
\hline Year 3 to baseline & 527 & -0.47 & $(0.78)$ & 493 & -0.04 & $(0.68)$ & -0.42 & $(0.05)$ & $<0.01$ \\
\hline \multicolumn{10}{|l|}{ HDL (mmol/l) } \\
\hline Baseline & 696 & 1.49 & $(0.37)$ & 661 & 1.48 & $(0.37)$ & 0.01 & $(0.02)$ & 0.72 \\
\hline Year 1 to baseline & 632 & 0.11 & $(0.23)$ & 596 & 0.01 & $(0.19)$ & 0.10 & $(0.01)$ & $<0.01$ \\
\hline Year 3 to baseline & 541 & 0.09 & $(0.25)$ & 506 & -0.03 & $(0.23)$ & 0.12 & $(0.02)$ & $<0.01$ \\
\hline \multicolumn{10}{|c|}{ Systolic BP (mm Hg) } \\
\hline Baseline & 8014 & 127.21 & $(17.51)$ & 7627 & 127.33 & $(17.41)$ & -0.13 & $(0.28)$ & 0.65 \\
\hline Year 1 to baseline & 7517 & -1.13 & $(15.07)$ & 7180 & -2.26 & $(15.30)$ & 1.14 & $(0.25)$ & $<0.01$ \\
\hline Year 3 to baseline & 7103 & -0.13 & $(17.01)$ & 6737 & -1.71 & (16.91) & 1.58 & $(0.29)$ & $<0.01$ \\
\hline \multicolumn{10}{|c|}{ Diastolic BP (mm Hg) } \\
\hline Baseline & 8014 & 75.64 & $(9.11)$ & 7627 & 75.82 & $(9.05)$ & -0.17 & $(0.15)$ & 0.23 \\
\hline Year 1 to baseline & 7517 & -1.44 & $(8.52)$ & 7179 & -1.40 & $(8.61)$ & -0.04 & $(0.14)$ & 0.79 \\
\hline Year 3 to baseline & 7101 & -1.73 & $(9.42)$ & 6739 & -1.94 & $(9.54)$ & 0.21 & $(0.16)$ & 0.20 \\
\hline \multicolumn{10}{|c|}{ Body Mass Index $\left(\mathrm{kg} / \mathrm{m}^{2}\right)$} \\
\hline Baseline & 7978 & 28.23 & $(5.70)$ & 7577 & 28.29 & $(5.81)$ & -0.07 & $(0.09)$ & 0.46 \\
\hline Year 1 to baseline & 7406 & -0.21 & $(2.70)$ & 7076 & -0.02 & $(2.41)$ & -0.19 & $(0.04)$ & $<0.01$ \\
\hline Year 3 to baseline & 7000 & 0.30 & $(2.93)$ & 6633 & 0.36 & $(2.74)$ & -0.06 & $(0.05)$ & 0.23 \\
\hline \multicolumn{10}{|c|}{ Waist circumference $(\mathrm{cm})$} \\
\hline Baseline & 7990 & 87.40 & (13.3) & 7601 & 87.50 & (13.6) & -0.13 & $(0.22)$ & 0.56 \\
\hline Year 1 to baseline & 7331 & -0.70 & $(6.3)$ & 7033 & 0.10 & $(6.2)$ & -0.77 & $(0.10)$ & $<0.01$ \\
\hline Year 3 to baseline & 3055 & 0.50 & $(7.6)$ & 2860 & 1.10 & $(7.1)$ & -0.58 & $(0.19)$ & $<0.01$ \\
\hline
\end{tabular}

a Statistics for blood analyte variables are weighted by race/ethnicity. ${ }^{\mathrm{b}}$ The $p$ values are from two-sample $t$ tests. Abbreviations: $\mathrm{BP}$, blood pressure; HOMA-IR, homeostasis model assessment of insulin resistance 
Table 3. Self-reported diabetes incidence by treatment assignment overall and in subgroups

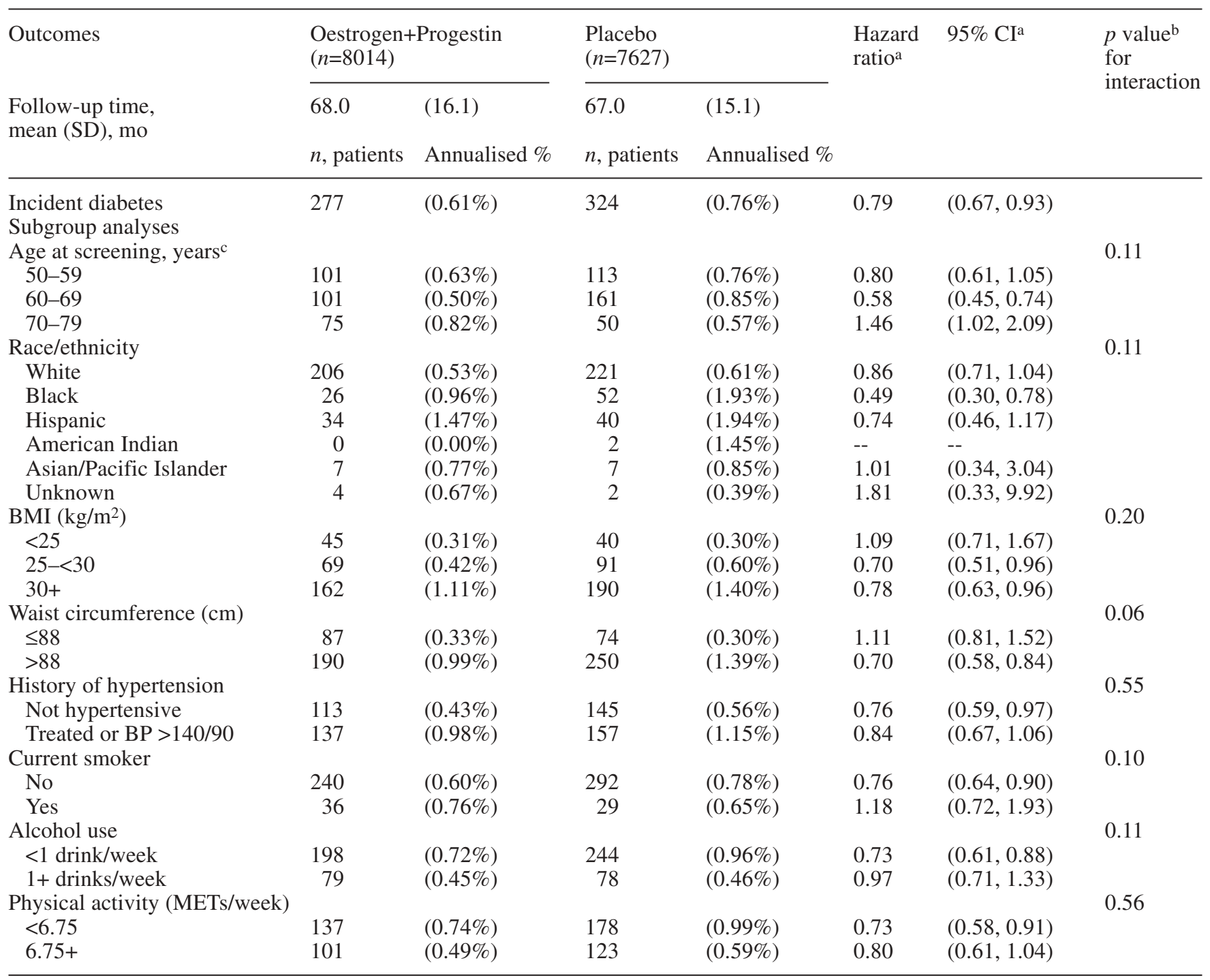

a Hazard ratios and confidence intervals (CIs) are from Cox proportional hazards analyses stratified by age and randomisation status in the low-fat diet trial. $\mathrm{b}$ The $p$ values are from a likelihood ratio test comparing a model with the main effects for the variable of interest and intervention group versus a model with the main effects plus the interaction between the main effects. ${ }^{c}$ Age subgroup models are only stratified by randomisation status in the diet modification trial. BP, blood pressure; MET, metabolic equivalent of the task who had impaired fasting glucose (based on a single fasting glucose measurement of $5.6-6.9 \mathrm{mmol} / \mathrm{l}$ ) at baseline, significant decreases were also noted between baseline and Year 1 for actively treated women compared to control subjects. These decreases were as follows: glucose $(-0.16 \pm 0.06 \mathrm{mmol} / \mathrm{l}, p=0.01)$; insulin $(-1.47 \pm 0.74 \mathrm{mU} / 1, p=0.05)$; HOMA-IR $(-0.50 \pm 0.22$, $p=0.02)$.

After one year, there was no significant difference in triglyceride levels between the two groups of women. Total cholesterol and LDL fell significantly, while HDL rose in the active treatment group compared with the control subjects. Compared to placebo, systolic blood pressure was about $1 \mathrm{~mm} \mathrm{Hg}$ higher $(p<0.01)$ in the oestrogen plus progestin group after one year of follow-up, but there was no difference in diastolic blood pressure. BMI fell by $0.21 \mathrm{~kg} / \mathrm{m}^{2}$ in the oestrogen plus progestin group and remained unchanged in the placebo group, resulting in a significant difference in the change in BMI between Year 1 and baseline $(-0.19 \pm 0.04, p<0.01)$. Waist circumference also decreased in the oestrogen plus progestin group (Year 1 to baseline between-group difference $-0.77 \pm 0.10 \mathrm{~cm}, p<0.01)$.

By Year 3, when the sample size was somewhat smaller and $37 \%$ of the women were non-adherent to the study medication, some of the differences described above (glucose, HOMA-IR and BMI) were smaller and no longer statistically significant when analysed according to intention-to-treat (Table 2). 


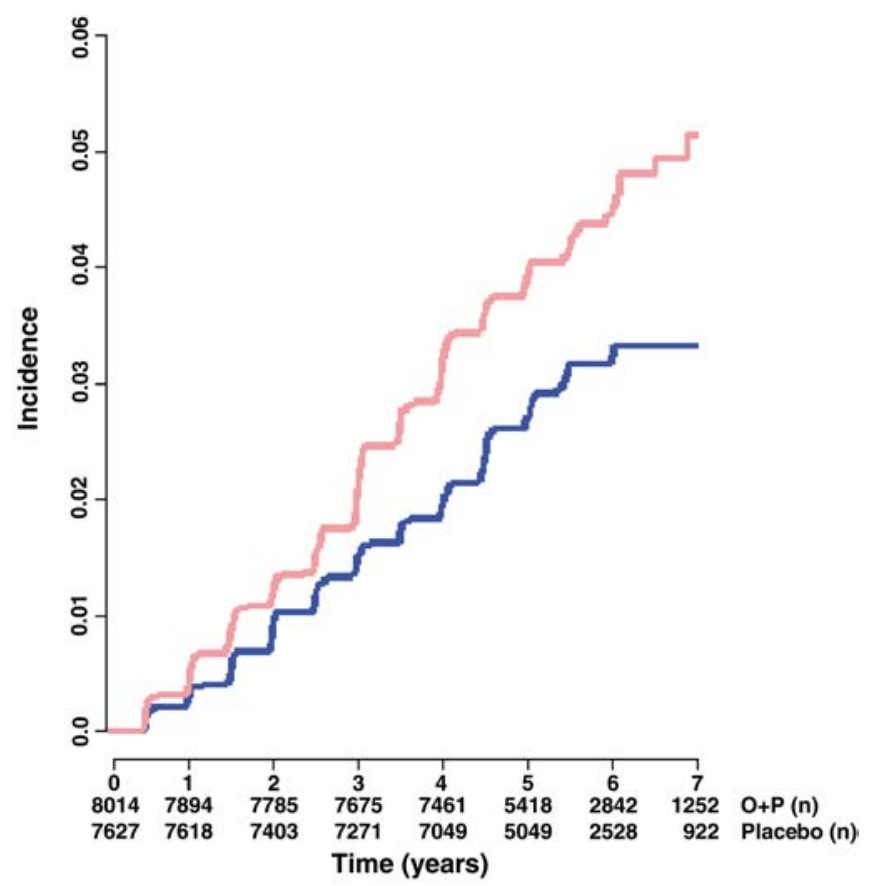

Fig. 1. Diabetes incidence by treatment arm (Oestrogen Plus Progestin $[\mathrm{O}+\mathrm{P}]$ versus Placebo). Hazard ratio $(95 \% \mathrm{CI})$, 0.79 (0.67-0.93). Blue line: Oestrogen Plus Progestin; red line: Placebo

When only the 748 women who were adherent at Year 3 were analysed, the results for glucose (Year 3 to baseline between-group difference $-0.07 \pm 0.07$, $p=0.32$, data not shown) and HOMA-IR (Year 3 to baseline between-group difference $-0.28 \pm 0.15$, $p=0.07$, data not shown) were quite similar to the intention-to-treat data. However, among 4560 adherent participants in the active treatment group and 4968 adherent participants in the control group who had measurements both at baseline and Year 3, BMI rose by 0.26 and 0.38 respectively (Year 3 to baseline between-group difference $-0.12 \pm 0.06, p=0.04$, data not shown).

As shown in Table 3 and Figure 1, after an average of 5.6 years of follow-up, the risk of developing diabetes was lower in the oestrogen plus progestin treatment group than in the placebo group. There were 277 cases of self-reported treated diabetes in the intervention group (cumulative incidence $3.5 \%$, annualised incidence $0.61 \%$ ), compared with 324 cases in the placebo group (cumulative incidence $4.2 \%$, annualised incidence $0.76 \%$ ). This represents an absolute reduction of 15 cases of incident treated diabetes per 10,000 women per year of treatment, and a $21 \%$ relative reduction in the risk of incident treated diabetes (HR 0.79, nominal 95\% CI 0.67-0.93). Thus to prevent one case of diabetes over 5.6 years, 143 women would have to be treated with oestrogen plus progestin. We also analysed the rate of incident diabetes (either by self-report, or by a glucose level of $\geq 7.0 \mathrm{mmol} / \mathrm{l}$ at Year 1 or Year 3) in the subsample of 1317 women

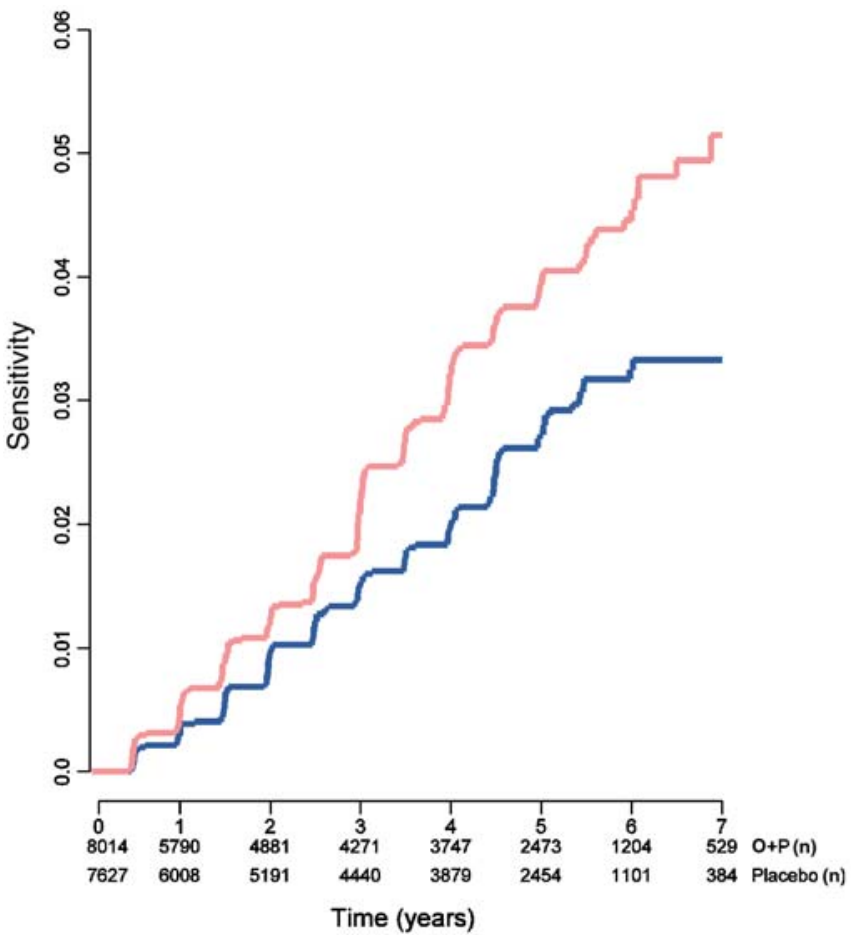

Fig. 2. Sensitivity analysis of diabetes in adherent participants by treatment arm. Participants were censored 6 months after becoming non-adherent (defined as taking less than $80 \%$ of study medication or starting open-label hormone therapy). Hazard ratio $(95 \%$ CI), 0.67 (0.54-0.82). Blue line: Oestrogen Plus Progestin; red line: Placebo

who had fasting glucose levels of less than $7.0 \mathrm{mmol} / \mathrm{l}$ at baseline. The point estimate of the hazard ratio (0.78) was nearly identical, but the result was not significant due to the much smaller sample size $(95 \% \mathrm{CI}$ $0.49-1.26, p=0.31$ ).

The sensitivity analysis (Fig. 2) shows that when participants who took less than $80 \%$ of study pills or began therapy with open-label hormones were censored, the magnitude of the difference between the groups was larger, with a $33 \%$ reduction in the risk of incident treated diabetes in the group receiving active hormone treatment (HR 0.67, 95\% CI 0.54-0.82). In comparison with women who took $80 \%$ or more of the placebo pills, women who took at least $80 \%$ of the active hormone pills had similar glucose levels, race/ ethnicity, BMI, waist circumference, and physical activity at baseline. However, there were some minor differences between adherent women in the two groups: adherent women on active hormone pills were 0.6 years younger, $2 \%$ more likely to have used hormone therapy before the trial, had lower blood pressure (1 mm Hg lower), had $146 \mathrm{~kJ} /$ day higher caloric intake, and were $2 \%$ less likely to use aspirin regularly. The sensitivity analysis results shown in Figure 2 were unchanged by adjustment for these variables (HR 0.68, 95\% CI 0.55-0.84).

In analyses of subgroups with known risk factors for diabetes, $p$ values in formal tests for interaction 
Table 4. Self-reported diabetes incidence by treatment assignment, adjusted for age, baseline BMI and change in BMI

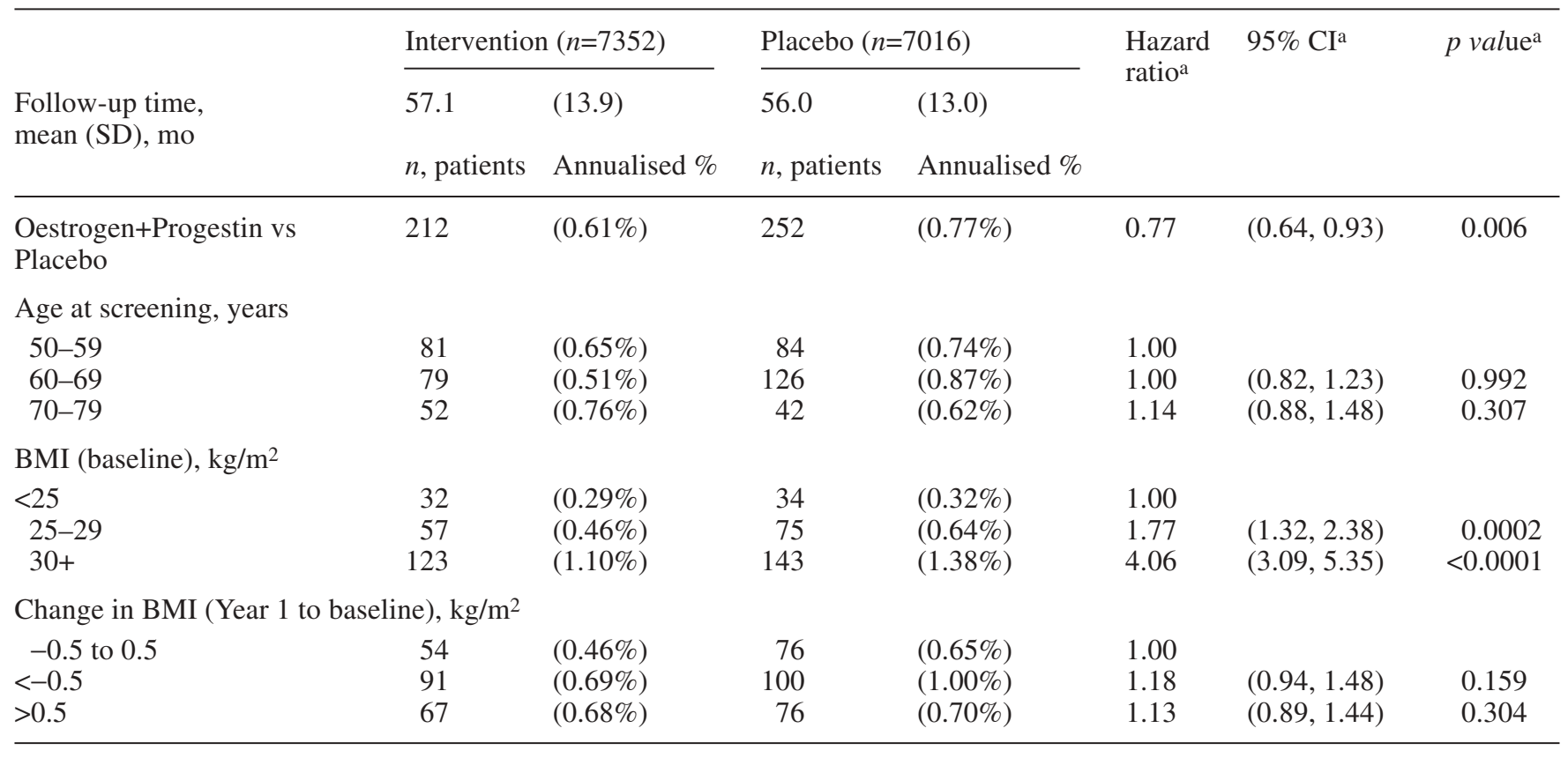

a Hazard ratios, confidence intervals (CIs) and $p$ values are from Cox proportional hazards analyses stratified by randomisation status in the diet modification trial

Table 5. Self-reported diabetes incidence by treatment assignment adjusted for age, baseline waist circumference and change in waist circumference

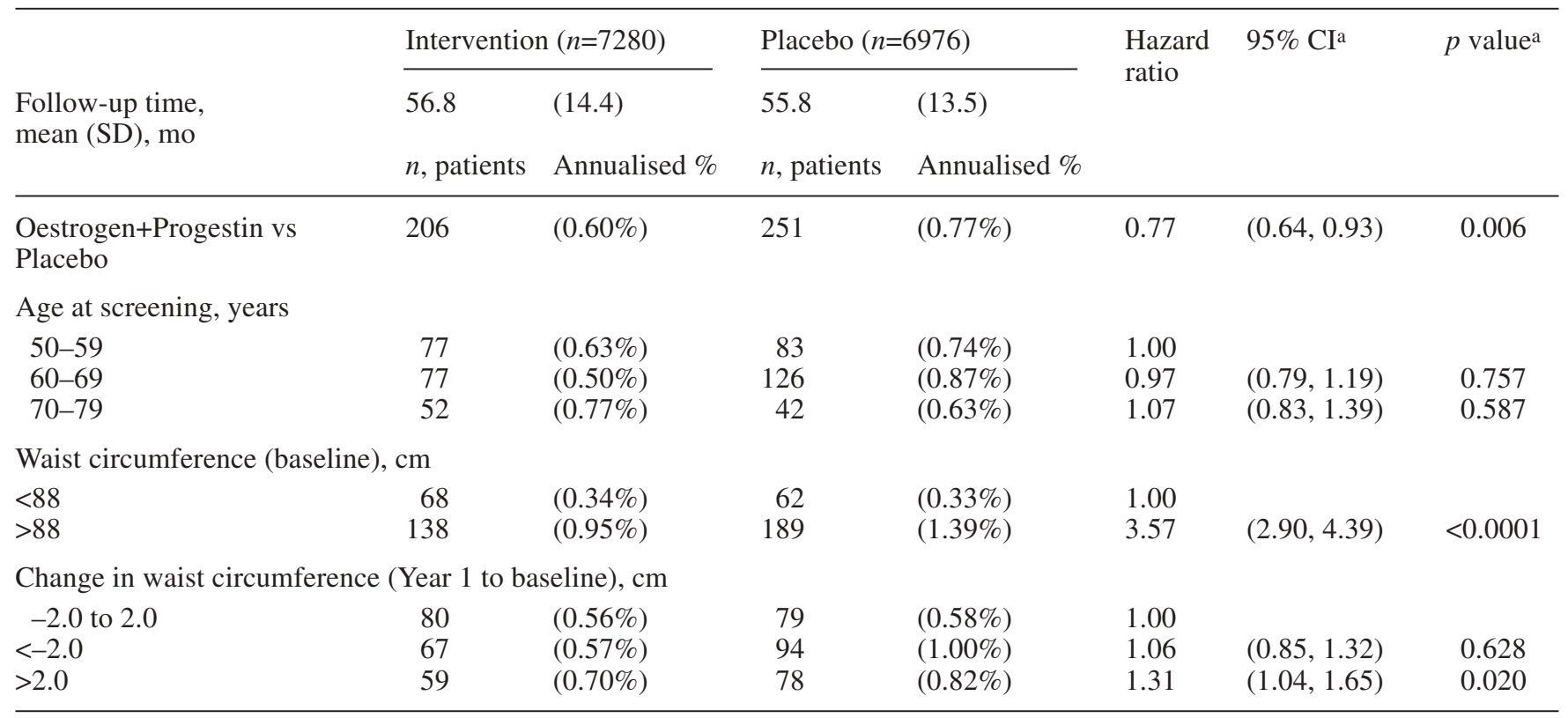

a Hazard ratios, confidence intervals (CIs) and $p$ values are from Cox proportional hazards analyses stratified by randomisation status in the low-fat diet trial

were greater than 0.05 for all subgroups (Table 3). There was a trend for women with a waist circumference of $88 \mathrm{~cm}$ or less not to derive as much protection from incident diabetes with oestrogen plus progestin treatment as women with larger waist circumferences at baseline (HR 1.11 vs HR 0.70, $p$ for interaction = 0.06). However, this finding may have been due to chance because of the number of subgroups examined.

To determine whether treatment-related favourable changes in BMI and waist circumference accounted for the observed protection from diabetes in the oestrogen plus progestin group, we further examined tertiles of change after one year in these variables in 
two separate age-adjusted multivariable Cox proportional hazards models, including baseline BMI in the first model and baseline waist circumference in the second (Tables 4 and 5). There was little change in the HR for active treatment $(0.77$, 95\% CI $0.64-0.93)$ after adjustment for change in BMI or change in waist circumference, with both models resulting in the same HR and 95\% CI. Baseline obesity and large waist circumference were both strongly associated with incident diabetes after accounting for age and treatment effects, with three- to four-fold elevation in risk. An increase in waist circumference of more than $2 \mathrm{~cm}$ in the first year of the study was weakly associated with risk of diabetes, after adjustment for age, treatment and baseline waist circumference.

\section{Discussion}

Our results show that treatment with combined oestrogen and progestin resulted in a lower incidence of self-reported treatment for diabetes in predominantly healthy women. As with the findings of the HERS study [26], the hazard ratio remained significant and unchanged even after adjusting for the small changes in BMI and waist circumference associated with active treatment. We found that oestrogen plus progestin lowered fasting glucose, fasting insulin and HOMAIR after one year. There were also favourable changes in lipids. However, the number of participants with these blood measurements was too small to rigorously determine whether these favourable metabolic changes accounted for the protection from diabetes associated with oestrogen plus progestin treatment.

Previous research examining the effect of hormone therapy on risk factors for and on development of diabetes has been inconclusive, and no long-term randomised clinical trial results were available until the recently published HERS findings [26]. The WHI and HERS trial findings concur with the observational findings of the large Nurses Health Study $(n=21,028)$, where current users of hormone therapy had a reduced incidence of diabetes (relative risk 0.80, 95\% CI 0.67-0.96) when compared with past users and women who had never used hormone therapy and whose values were adjusted for age and BMI [25].

Our data show a small but significant decrease in fasting glucose $(0.12 \mathrm{mmol} / \mathrm{l})$ and fasting insulin $(0.65 \mathrm{mU} / \mathrm{l})$ after 1 year on oestrogen plus progestin compared to placebo. Many investigators have examined the effect of hormone therapy on intermediate outcomes such as glucose and insulin levels, and responses to glucose tolerance tests, with varying results. The largest of these intermediate outcome trials was the Postmenopausal Estrogen/Progesterone Interventions trial, which tested four different regimens containing conjugated equine oestrogens against placebo. This study found lower fasting glucose levels but high- er 2-hour post-challenge levels with all active hormone regimens [2]. Similar findings were seen in several other trials using conjugated equine oestrogens $[4,5$, $10]$ and in several large observational studies [23, 33, 34]. Other experimental and observational studies failed to find significant differences in glucose levels between hormone therapy users and non-users [7, 9, $14,16,17,18,19,20,35,36,37,38,39,40,41,42$, $43,44]$ or found elevated levels of fasting glucose [12, 13]. Likewise, some studies have found lower levels of fasting insulin in hormone users $[2,4,5,6,7,8,9,33$, $35,41,43,45]$, but others have found no change [10, $14,16,17,18,19,20,23,34,36,37,42,44]$.

Using the HOMA-IR calculation to estimate insulin resistance, we found a small but significant decrease in insulin resistance in the oestrogen plus progestin group. Insulin sensitivity in women taking oestrogen has been examined previously, with some studies pointing to improved insulin sensitivity [37, 39, 41] and better rates of glucose disposal [46] among women who use oestrogen. This finding, however, has not been consistent $[6,11,15,16,17,20,38,42$, 44].

Our data show a small but statistically significant decrease in BMI and waist circumference during the first year of treatment with oestrogen plus progestin, although the BMI differences lessened during further follow-up. Several previous studies have found improvements in BMI [38], fat mass [38, 41] and waistto-hip ratios [38] with combined hormone therapy. Others have found no change. [9, 19, 20, 39, 44]. The HERS study found nearly identical changes in BMI and waist circumference to those observed in the WHI, and also concluded that these changes were not mediators of the reduction in diabetes incidence observed in that study [26]. Some of the variation in the results in intermediate endpoints reviewed above may be due to the following: (i) the small number of women included [4, 6, 11, 13, 14, 16, 17, 18, 20, 37, 38]; (ii) the short duration of the trial $[6,16,17,20,42$, 44]; (iii) the use of a higher dose of conjugated equine oestrogens $[11,12,13,16,37]$; or (iv) the use of another type of oestrogen $[6,7,8,9,16,17,19,20$, $24,38,42,43,44]$ or progestin $[8,14,15,17,19]$.

Even though the changes in glucose and insulin noted in our study at one year were small, taken together, they amount to a significant decrease in estimated insulin resistance. However, at 3 years, the glucose difference was much smaller, and not significant, while the HOMA-IR difference was of a similar magnitude, but with borderline significance. The lower adherence at Year 3 does not appear to explain these findings. Although our results suggest that lower insulin resistance may have been responsible for the lower incidence of diabetes observed in the women who received active treatment, further investigation of this and other potential mechanisms is needed.

Of the many human and animal studies that have investigated the link between sex steroids and insulin 
action, few have involved postmenopausal women [47]. In a prospective study of older women who were not using hormone therapy, endogenous levels of bioavailable oestradiol and testosterone were positively associated with 8-year follow-up levels of fasting insulin and calculated HOMA-IR, while only bioavailable testosterone predicted incident diabetes [48]. Positive associations of bioavailable oestradiol and testosterone with insulin resistance have also been observed in a cross-sectional study of untreated postmenopausal women [49]. Low levels of sex hormone binding globulin (SHBG) are related to both insulin resistance and incident diabetes in postmenopausal women $[50,51,52]$. In a small placebo-controlled crossover trial in postmenopausal women with Type 2 diabetes, the administration of combined oestrogen plus progestin increased SHBG and decreased free testosterone [53]. Thus, to understand the mechanisms by which exogenous administration of oestrogen plus progestin decreases insulin resistance and the development of diabetes in postmenopausal women, it will probably be necessary to define the complex interplay between gonadotropins, adrenal hormones, ovarian hormones, SHBG and adipose tissue.

Some limitations of our study design must be acknowledged. Our outcome was based on self-reports of diabetes treatment with oral medications or insulin. In population-based studies in the US and Europe, a self-report of Type 2 diabetes mellitus was confirmed in as low as $64 \%$ and as high as $96 \%$ of cases [54, 55, $56,57,58]$, but no study has looked at the validity of a case definition similar to that for treated diabetes in the WHI. At baseline, $79 \%$ of participants in the treatment and control groups of the WHI Estrogen plus Progestin Trial who self-reported treatment with diabetes medication actually had an oral hypoglycaemic medication or insulin recorded in the baseline medication inventory (unpublished data on participants excluded from this analysis). It is therefore unlikely that over-reporting of diabetes treatment substantially affected our results. However, many cases of incident diabetes are treated initially with lifestyle intervention, and our method of case ascertainment would not have detected these. In addition, a substantial proportion of diabetes is undiagnosed. Our baseline glucose data in participants without a physician-diagnosed history of diabetes shows that about $3 \%$ had undiagnosed diabetes based on fasting glucose of $7.0 \mathrm{mmol} / \mathrm{l}$ or higher. In population-based studies in the United States in the early to mid-1990s, the proportion of undiagnosed diabetes based on the fasting glucose criterion in post-menopausal women was slightly higher at about 5\% [59]. The WHI design did not include the use of fasting or post-challenge hyperglycaemia at follow-up to find cases of undiagnosed diabetes. Thus, our data do not address the effect of oestrogen plus progestin on undiagnosed diabetes or diabetes treated with lifestyle interventions alone.
At baseline, $27 \%$ of the participants had impaired fasting glucose $(5.6-6.9 \mathrm{mmol} / \mathrm{l})$, but there were too few events in the relatively small subsample with baseline glucose measurements for us to determine the effect of oestrogen plus progestin in these $27 \%$ of subjects with a very high risk of progression to diabetes. The same limitation applies to participants who may have fulfilled other criteria for the metabolic syndrome, e.g. abnormal values for triglycerides and HDL cholesterol. Our data do not address the effect of oestrogen alone on diabetes incidence, which will be examined in the recently halted WHI trial of oestrogen alone vs placebo [60]. The role of progestin in carbohydrate metabolism has not been fully explored; however, it is unlikely to account for our results. In fact, other studies have pointed to a detrimental effect of medroxyprogesterone acetate both on incidence of diabetes [61] and on carbohydrate metabolism [37]. Finally, a wide range of clinical and behavioral outcomes have been examined in the Estrogen plus Progestin section of the WHI Hormone Trial, increasing the probability that some outcomes may be statistically significant owing to chance alone.

In summary, these data suggest that combined therapy with oestrogen and progestin reduces the incidence of diabetes, possibly mediated by a decrease in insulin resistance unrelated to body size. In view of the overall adverse effects of conjugated equine oestrogens plus medroxyprogesterone acetate found in the Women's Health Initiative Hormone Trial [27], our findings do not justify the use of combined oestrogen plus progestin therapy for the prevention of diabetes in postmenopausal women. However, further study of mechanisms seems warranted to explore the role of hormonal factors in diabetes prevention. Future studies of alternative postmenopausal hormone therapy regimens and selective oestrogen agonists and/or antagonists should consider the effects of these regimens on insulin resistance and diabetes.

Acknowledgements. The WHI programme is funded by the National Heart, Lung and Blood Institute, US Department of Health and Human Services. Dr K.L. Margolis received support through an award from the National Heart, Lung, and Blood Institute (K23 HL03996). Dr L.S. Phillips received support through awards from National Institute of Diabetes, Digestive and Kidney Diseases, and the Agency for Healthcare Research and Quality (HS-07922). J.I. Torréns was supported by NIH HD 01457 as a BIRCWH Scholar.

\section{Appendix}

The Women's Health Initiative Research Group

Program Office: (National Heart, Lung, and Blood Institute, Bethesda, Md., USA) Alving B, Rossouw J, Pottern L 
Clinical Coordinating Center: Fred Hutchinson Cancer Research Center, Seattle, Wash., USA:

Prentice RL, Anderson G, LaCroix A, Patterson RE, McTiernan A; Wake Forest University School of Medicine, Winston-Salem, N.C., USA: Shumaker S, Rautaharju P; Medical Research Labs, Highland Heights, Ky., USA: Stein E; University of California at San Francisco, San Francisco, Calif., USA: Cummings S; University of Minnesota, Minneapolis, Minn., USA: Himes J; University of Washington, Seattle, Wash., USA: Psaty B.

Clinical Centers: Albert Einstein College of Medicine, Bronx, N.Y., USA: Wassertheil-Smoller S; Baylor College of Medicine, Houston, Tex., USA: Hays J; Brigham and Women's Hospital, Harvard Medical School, Boston, Mass., USA: Manson J; Brown University, Providence, R.I., USA: Assaf AR; Emory University, Atlanta, Ga., USA: Phillips L; Fred Hutchinson Cancer Research Center, Seattle, Wash., USA: Beresford S; George Washington University Medical Center, Washington, D.C., USA: Hsia J; HarborUCLA Research and Education Institute, Torrance, Calif., USA: Chlebowski R; Kaiser Permanente Center for Health Research, Portland, Ore., USA: Ritenbaugh C; Kaiser Permanente Division of Research, Oakland, Calif., USA: Caan B; Medical College of Wisconsin, Milwaukee, Wis., USA: Kotchen JM; MedStar Research Institute/Howard University, Washington, D.C., USA: Howard BV; Northwestern University, Chicago/Evanston, Ill., USA: Van Horn L; Rush-Presbyterian St. Luke's Medical Center, Chicago, Ill., USA: Black H; Stanford Center for Research in Disease Prevention, Stanford University, Stanford, Calif., USA: Stefanick ML; State University of New York at Stony Brook, Stony Brook, N.Y., USA: Lane D; The Ohio State University, Columbus, Ohio, USA: Jackson R; University of Alabama at Birmingham, Birmingham, Ala., USA: Lewis CB; University of Arizona, Tucson/Phoenix, Ariz., USA: Bassford T; University at Buffalo, Buffalo, N.Y., USA: WactawskiWende J; University of California at Davis, Sacramento, Calif., USA: Robbins J; University of California at Irvine, Orange, Calif., USA: Hubbell A; University of California at Los Angeles, Los Angeles, Calif., USA: Judd H; University of California at San Diego, LaJolla/ Chula Vista, Calif., USA: Langer RD; University of Cincinnati, Cincinnati, Ohio, USA: Gass M; University of Florida, Gainesville/Jacksonville, Fla, USA: Limacher M; University of Hawaii, Honolulu, Hawaii, USA: Curb D; University of Iowa, Iowa City/Davenport, Iowa, USA: Wallace R; University of Massachusetts/Fallon Clinic, Worcester, Mass., USA: Ockene J; University of Medicine and Dentistry of New Jersey, Newark, N.J., USA: Lasser N; University of Miami, Miami, Fla., USA: O'Sullivan MJ; University of Minnesota, Minneapolis, Minn., USA: Margolis K; University of Nevada, Reno, Nev., USA: Brunner R; University of North Carolina, Chapel Hill,
N.C., USA: Heiss G; University of Pittsburgh, Pittsburgh, Pa., USA: Kuller L; University of Tennessee, Memphis, Tenn., USA: Johnson KC; University of Texas Health Science Center, San Antonio, Tex., USA: Brzyski R; University of Wisconsin, Madison, Wis., USA: Sarto G; Wake Forest University School of Medicine, Winston-Salem, N.C., USA: Bonds D; Wayne State University School of Medicine/Hutzel Hospital, Detroit, Mich., USA: Hendrix S

\section{References}

1. Mokdad A, Ford E, Bowman B et al. (2003) Prevalence of obesity, diabetes and obesity-related health risk factors, 2001. JAMA 289:76-79

2. Espeland M, Hogan P, Fineberg S et al. (1998) Effect of postmenopausal hormone therapy on glucose and insulin concentrations. Diabetes Care 21:1589-1595

3. Writing group for the PEPI Trial (1995) Effects of estrogen or estrogen/progestin regimens on heart disease risk factors in postmenopausal women. The Postmenopausal Estrogen/ Progestin Interventions (PEPI) Trial. JAMA 273:199-208

4. Cagnacci A, Soldani R, Carriero PL, Paoletti AM, Fioretti P, Melis GB (1992) Effects of low doses of transdermal 17 beta-estradiol on carbohydrate metabolism in postmenopausal women. J Clin Endocrinol Metab 74:1396-1400

5. Lobo R, Pickar J, Wild R, Walsh B, Hirvonen E (1994) Metabolic impact of adding medroxyprogesterone acetate to conjugated estrogen therapy in postmenopausal women. Obstet Gynecol 84:987-995

6. Cagnacci A, Tuveri F, Cirillo R, Setteneri AM, Melis GB, Volpe A (1997) The effect of transdermal 17-beta-estradiol on glucose metabolism of postmenopausal women is evident during the oral but not the intravenous glucose administration. Maturitas 28:163-167

7. Colacurci N, Zarcone R, Mollo A et al. (1998) Effects of hormone replacement therapy on glucose metabolism. Panminerva Medica 40:18-21

8. Davidson M, Maki K, Marx P et al. (2000) Effects of continuous estrogen and estrogen-progestin replacement regimens on cardiovascular risk markers in postmenopausal women. Arch Intern Med 160:3315-3325

9. Hodis H, Mack W, Lobo R et al. (2001) Estrogen in the prevention of atherosclerosis. Ann Intern Med 135:939953

10. Lobo R, Bush T, Carr B, Pickar J (2001) Effects of lower doses of conjugated equine estrogens and medroxyprogesterone acetate on plasma lipids and lipoproteins, coagulation factors, and carbohydrate metabolism. Fertil Steril 76:13-24

11. Goldman J, Ovadia J (1969) The effect of estrogen on intravenous glucose tolerance in women. Am J Obstet Gynecol 103:172-178

12. Spellacy W, Buhi W, Birk S (1972) The effect of estrogen on carbohydrate metabolism: glucose, insulin, and growth hormone studies on one hundred and seventy-one women ingesting Premarin, mestranol, and ethinyl estradiol for six months. Am J Obstet Gynecol 114:378-392

13. Ajabor L, Tsai C, Vela P, Yen S (1972) Effect of exogenous estrogen on carbohydrate metabolism in postmenopausal women. Am J Obstet Gynecol 113:383-387

14. De Cleyn K, Buytaert P, Coppens M (1989) Carbohydrate metabolism during hormonal substitution therapy. Maturitas 11:235-242 
15. Godsland I, Gangar K, Walton C et al. (1993) Insulin resistance, secretion, and elimination in postmenopausal women receiving oral and transdermal hormone replacement therapy. Metabolism 42:846-853

16. O'Sullivan A, Ho K (1995) A comparison of the effects of oral and transdermal estrogen replacement on insulin sensitivity in postmenopausal women. J Clin Endocrinol Metab 80:1783-1788

17. Kimmerle R, Heinemann L, Heise T et al. (1999) Influence of continuous combined estradiol-norethisterone acetate preparations on insulin sensitivity in postmenopausal nondiabetic women. Menopause 6:36-42

18. Roo G, Stehouwer C, Meijer P et al. (1999) Both raloxifene and estrogen reduce major cardiovascular risk factors in healthy postmenopausal women: a 2-year, placebo-controlled study. Arterioscler Thromb Vasc Biol 19:2993-3000

19. Seed M, Sands R, McLaren M, Kirk G, Darko D (2000) The effect of hormone replacement therapy and route of administration on selected cardiovascular risk factors in post-menopausal women. Fam Pract 17:497-507

20. Vehkavaara S, Westerbacka J, Hakala-Ala-Pietila T, Virkamaki A, Hovatta O, Yki-Jarvinen H (2000) Effect of estrogen replacement therapy on insulin sensitivity of glucose metabolism and preresistance and resistance vessel function in healthy postmenopausal women. J Clin Endocrinol Metab 85:4663-4670

21. Hammond CB, Jelovsek FR, Lee KL, Creasman WT, Parker RT (1979) Effects of long-term estrogen replacement therapy. I. Metabolic effects. Am J Obstet Gynecol 133:525-536

22. Gabal L, Goodman-Gruen D, Barrett-Connor E (1997) The effect of postmenopausal estrogen therapy on the risk of non-insulin-dependent diabetes mellitus. Am J Pub Health 87:443-445

23. Zhang Y, Howard B, Cowan L et al. (2002) The effect of estrogen use on levels of glucose and insulin and the risk of type 2 diabetes in American Indian postmenopausal women: the Strong Heart Study. Diabetes Care 25:500-504

24. Rossi R, Origlinani G, Modena M (2004) Transdermal 17-beta-estradiol and risk of developing type 2 diabetes in a population of healthy, nonobese postmenopausal women. Diabetes Care 27:645-649

25. Manson J, Rimm E, Colditz G et al. (1992) A prospective study of postmenopausal estrogen therapy and subsequent incidence of non-insulin-dependent diabetes mellitus. Ann Epidemiol 2:665-673

26. Kanaya A, Herrington D, Vittinghoff E et al. (2003) Glycemic effects of postmenopausal hormone therapy: The Heart and Estrogen/progestin Replacement Study. Ann Intern Med 138:1-9

27. Women's Health Initiative Study Group (1998) Design of the Women's Health Initiative Clinical Trial and Observational Study. Control Clin Trials 19:61-109

28. Women's Health Initiative Investigators (2002) Risks and benefits of estrogen plus progestin in healthy postmenopausal women: Principal results from the Women's Health Initiative randomized controlled trial. JAMA 288:321-333

29. Block G, Woods M, Potosky A, Clifford C (1990) Validation of a self-administered diet history questionnaire using multiple diet records. J Clin Epidemiol 43:1327-1335

30. Patterson R, Kristal A, Coates R et al. (1996) Low-fat diet practices of older women: prevalence and implications for dietary assessment. J Am Diet Assoc 96:670-679

31. Ainsworth B, Haskell W, Leon A et al. (1993) Compendium of physical activities: Classification of energy costs of human physical activities. Med Sci Sports Exerc 25:7180
32. Matthews D, Hosker J, Rudenski A, Naylor B, Treacher D, Turner R (1985) Homeostasis model assessment: insulin resistance and B-cell function from fasting plasma glucose and insulin concentrations in man. Diabetologia 28:412419

33. Nabulsi A, Folsom A, White A et al. (1993) Association of hormone replacement therapy with various cardiovascular risk factors in postmenopausal women. $\mathrm{N}$ Engl J Med 328:1069-1075

34. Troisi R, Cowie C, Harris M (2000) Hormone replacement therapy and glucose metabolism. Obstet Gynecol 96:665670

35. Barrett-Connor E, Laakso M (1990) Ischemic heart disease risk in postmenopausal women: effects of estrogen use on glucose and insulin levels. Arteriosclerosis 10:531-534

36. Cucinelli F, Paparella P, Soranna L et al. (1999) Differential effect of transdermal estrogen plus progestagen replacement therapy on insulin metabolism in postmenopausal women: relation to their insulinemic secretion. Eur $\mathrm{J}$ Endocrinol 140:215-223

37. Lindheim SR, Presser SC, Ditkoff EC, Vijod MA, Stanczyk FZ, Lobo RA (1993) A possible bimodal effect of estrogen on insulin sensitivity in postmenopausal women and the attenuating effect of added progestin. Fertil Steril 60:664-667

38. Sites C, Brochu M, Tchernof A, Poehlman E (2001) Relationship between hormone replacement therapy use with body fat distribution and insulin sensitivity in obese postmenopausal women. Metabolism 50:835-840

39. Ryan A, Nicklas B, Berman D (2002) Hormone replacement therapy, insulin sensitivity, and abdominal obesity in postmenopausal women. Diabetes Care 25:127-133

40. Roussel A-M, Bureau I, Favier M, Polansky M, Bryden N, Anderson R (2002) Beneficial effects of hormone replacement therapy on chromium status and glucose and lipid metabolism in postmenopausal women. Maturitas 42:63-69

41. Evans E, Van Pelt E, Binder E, Williams D, Ehsani A, Kohrt W (2001) Effects of HRT and exercise training on insulin action, glucose tolerance, and body composition in older women. J Appl Physiol 90:2033-2040

42. Duncan A, Lyall H, Roberts R et al. (1999) The effect of estradiol and a combined estradiol/progestagen preparation on insulin sensitivity in healthy postmenopausal women. J Clin Endocrinol Metab 84:2402-2407

43. Crook D, Godsland I, Hull J, Stevenson J (1997) Hormone replacement therapy with dydrogesterone and 17 betaoestradiol: effects on serum lipoproteins and glucose tolerance during 24 month follow up. Br J Obstet Gynaecol 104:298-304

44. Walker RJ, Lewis-Barned NJ, Sutherland WH et al. (2001) The effects of sequential combined oral 17 beta-estradiol norethisterone acetate on insulin sensitivity and body composition in healthy postmenopausal women: a randomized single blind placebo-controlled study. Menopause 8:27-32

45. Fonseca E, Ochoa R, Galvan R, Hernandez M, Mercado M, Zarate A (1999) Increased serum levels of growth hormone and insulin-like growth factor-I associated with simultaneous decrease of circulating insulin in postmenopausal women receiving hormone replacement therapy. Menopause 6:56-60

46. Wilcox JG, Hwang J, Hodis HN, Sevanian A, Stanczyk FZ, Lobo RA (1997) Cardioprotective effects of individual conjugated equine estrogens through their possible modulation of insulin resistance and oxidation of low-density lipoprotein. Fertil Steril 67:57-62

47. Livingstone C, Collison M (2002) Sex steroids and insulin resistance. Clin Sci (Lond) 102:151-166 
48. Oh J-Y, Barrett-Connor E, Wedick N, Wingard D (2002) Endogenous sex hormones and the development of type 2 diabetes in older men and women: the Rancho Bernardo Study. Diabetes Care 25:55-60

49. Kalish G, Barrett-Connor E, Laughlin G, Gulanski B (2003) Association of endogenous sex hormones and insulin resistance among postmenopausal women: results from the Postmenopausal Estrogen/Progestin Intervention Trial. J Clin Endocrinol Metab 88:1646-1652

50. Haffner S, Dunn J, Katz MS (1992) Relationship of sexhormone-blinding globulin to lipid, lipoprotein, glucose and insulin concentrations in postmenopausal women. Metabolism 41:278-284

51. Lindstedt G, Lundberg P-A, Lapidus L, Lundgren H, Bengtsson B, Björntorp P (1991) Low sex-hormone-binding globulin concentration as independent risk factor for development of NIDDM. Diabetes 40:123-128

52. Haffner S, Valdez R, Morales P, Hazuda H, Stern M (1993) Decreased sex-hormone-binding globulin predicts noninsulin-dependent diabetes mellitus in women but not in men. J Clin Endocrinol Metab 77:56-60

53. Andersson B, Mattsson L, Hahn L et al. (1997) Estrogen replacement therapy decreases hyperandrogenicity and improves glucose homeostasis and plasma lipids in postmenopausal women with noninsulin-dependent diabetes mellitus. J Clin Endocrinol Metab 82:638-643

54. Kaye S, Folsom A, Sprafka J, Prineas R, Wallace R (1991) Increased incidence of diabetes mellitus in relation to abdominal adiposity in older women. J Clin Epidemiol 44:329-334
55. Kehoe R, Wu S-Y, Leske M, Chylack L (1994) Comparing self-reported and physician-reported medical history. Am J Epidemiol 139:813-818

56. Midthjell K, Holmen J, Bjorndal A, Lund-Larsen P (1992) Is questionnaire information valid in the study of a chronic disease such as diabetes? The Nord-Trondelag diabetes study. J Epidemiol Community Health 46:537-542

57. Tretli S, Lund-Larsen P, Foss O (1982) Reliability of questionnaire information on cardiovascular disease and diabetes: cardiovascular disease study in Finnmark county. J Epidemiol Community Health 36:269-273

58. Kriegsman D, Penninx B, Eijk J van, Boeke A, Deeg D (1996) Self-reports and general practitioner information on the presence of chronic diseases in community dwelling elderly: a study on the accuracy of patients' self-reports and on determinants of inaccuracy. J Clin Epidemiol 49:1407-1417

59. Harris M, Flegal K, Cowie C et al. (1998) Prevalence of diabetes, impaired fasting glucose, and impaired glucose tolerance in U.S. adults. The Third National Health and Nutrition Examination Study, 1988-1994. Diabetes Care 21:518-524

60. The Women's Health Initiative Steering Committee (2004) Effects of conjugated equine estrogens in postmenopausal women with hysterectomy: the Women's Health Initiative randomized controlled trial. JAMA 291:1701-1712

61. Kim C, Seidel K, Begier E, Kwok Y (2001) Diabetes and depot medroxyprogesterone contraception in Navajo women. Arch Intern Med 161:1766-1771 